\title{
Interactions between Yersinia pestis V- antigen (LCrV) and human Toll-like receptor 2 (TLR2) in a modelled protein complex and potential mechanistic insights
}

Tiandi Wei ${ }^{1,2}$, Jing Gong ${ }^{3}$, Guojing Qu ${ }^{2}$, Mingyu Wang ${ }^{1,2^{*}}$ (D) and Hai $X u^{1}$

\begin{abstract}
Background: Yersinia pestis, the etiological pathogen of plague, is capable of repressing the immune response of white blood cells to evade phagocytosis. The V-antigen (LCrV) was found to be involved in this process by binding to human Toll-like Receptor 2 (TLR2). The detailed mechanism behind this LcrV and TLR2 mediated immune response repression, however, is yet to be fully elucidated due to the lack of structural information.

Results: In this work, with protein structure modelling, we were able to construct a structure model of the heterotetramer of $Y$. pestis LcrV and human TLR2. Molecular dynamics simulation suggests the stability of this structure in aquatic environment. The LcrV model has a dumbbell-like structure with two globule domains (G1 at $\mathrm{N}$-terminus and G2 away from membrane) connected with a coiled-coil linker (CCL) domain. The two horseshoeshape TLR2 subunits form a V-shape structure, are not in direct contact with each other, and are held together by the LCrV homodimer. In this structure model, both the G1 and CCL domains are involved in the formation of LcrV homodimer, while all three domains are involved in LcrV-TLR2 binding. A mechanistic model was proposed based on this heterotetrameric structure model: The LcrV homodimer separates the TLR2 subunits to inhibit the dimerization of TLR2 and subsequent signal transfer for immune response; while LcrV could also inhibit the formation of heterodimers of TLR2 with other TLRs, and leads to immune response repression.
\end{abstract}

Conclusions: A heterotetrameric structure of $Y$. pestis LcrV and human TLR2 was modelled in this work. Analysis of this modelled structure showed its stability in aquatic environments and the role of LcrV domains and residues in protein-protein interaction. A mechanistic model for the role of LcrV in Y. pestis pathogenesis is raised based on this heterotetrameric structure model. This work provides a hypothesis of LcrV function, with which further experimental validation may elucidate the role of LcrV in human immune response repression.

Keywords: Yersinia pestis, LCrV, V-antigen, Toll-like receptor, TLR2, Plague, Structure modelling, Immune response repression

\section{Background}

Yersinia pestis is a deadly pathogen that caused three of the most catastrophic plagues in human history, including the notorious "Black Death" in Europe in Mid 1300 's, leading to the deaths of approximately 17 to 28 million people $[1,2]$. Today, despite extreme precautions

\footnotetext{
* Correspondence: wangmingyu@sdu.edu.cn

${ }^{1}$ State Key Laboratory of Microbial Technology, Microbial Technology Institute, Shandong University, Qingdao, China

${ }^{2}$ Taishan College, Shandong University, Qingdao, China

Full list of author information is available at the end of the article
}

that were taken in order to prevent the outbreak of $Y$. pestis, cases of $Y$. pestis infection that frequently result in patient deaths were still reported now and then [3]. Infection of $Y$. pestis is commonly mediated by bacteriacontaining aerosol inhalation or flea bite that transmits the bacterium from pathogen-carrying reservoir mammal hosts to human, leading to rapid progression of symptoms from fever to pneumonia, to hemoptysis, and eventually to patient deaths in 3-4 days $[4,5]$.

(c) The Author(s). 2019 Open Access This article is distributed under the terms of the Creative Commons Attribution 4.0 International License (http://creativecommons.org/licenses/by/4.0/), which permits unrestricted use, distribution, and 
One striking feature of $Y$. pestis is its ability to evade phagocytosis and grow in white blood cells such as macrophages [6]. This was done by injection of Yersinia outer membrane proteins (Yops) to cells by Type III Secretion System (T3SS, also termed the injectisome) upon contact with target cells $[7,8]$. The injected Yops subsequently repress phagocytosis and the immunity-related signal pathways [9]. Gene encoding these proteins reside on the virulence plasmid pYV (also termed pCD) that's co-hosted by a series of pathogenic Yersinia species such as Y. pestis, Yersinia pseudotuberculosis, and Yersinia enterocolitica [9-11]. Y. pseudotuberculosis and Y. enterocolitica are enteric members of the Yersinia genus that are transmitted primarily by contaminated food and water. These two species do not cause plagues but rather leads to a variety of diseases such as enterocolitis [12]. The pYV plasmid also carries a $l c r V$ gene that encodes a Low Calcium Response V (LcrV, also termed the Vantigen) protein. This protein has been considered important in the virulence of $Y$. pestis.

The role of LcrV in the pathogenesis of $Y$. pestis has been previously investigated in a variety of contradictory reports. LcrV was found secreted to the extracellular space to assist the entry of Yops to host cells [13, 14]. It was later found that LcrV leads to immune response repression by improving IL-10 expression and subsequently repressing inflammation factors TNF- $\alpha$ and IFN- $\gamma$ in $Y$. enterocolitica $[15,16]$. This response was found to be mediated by the binding of host Toll-like receptor 2 (TLR2) and LcrV at two independent binding sites (L32-L35 and D203-I206) [17-19]. Different signal transduction pathways were also proposed, suggesting that LcrV can repress TFN- $\alpha$ via a yet unknown IL-20 independent pathway [20]. However, in a report by Pouliot et al., controversy arose as the authors found $Y$. pestis TLR2 cannot be activated by LcrV and therefore is not able to mediate IL-10-dependent immune response by LcrV [21]. This finding was supported by a subsequent investigation showing $Y$. pestis $\mathrm{LcrV}$ cannot lead to significant IL-10 induction [22].

In order to further understand the role of LcrV in the pathogenesis of $Y$. pestis and the molecular mechanism by which LcrV represses immune response, structural information is needed for this protein, as well as for the interaction between this protein and its potential targets. The crystal structure of an entropy reduced mutant of $Y$. pestis LcrV was obtained at $2.2 \AA$ [1]. However, this structure was mutated at $\mathrm{K} 40-\mathrm{K} 42$, was incomplete at loop regions, and was monomeric despite reports suggesting LcrV is a homodimer [23]. Later attempts were able to solve the LcrV structure at $1.65 \AA$ [24]. This structure, however, is also incomplete for the lack of Cterminal loop structures. No investigations have been reported on the structure of the LcrV-TLR2 complex. This lack of structural knowledge prevents us from further elucidating the interaction of LcrV and TLR2, as well as further understanding the role of $\mathrm{LcrV}$ in $Y$. pestis pathogenesis.

In this work, aiming at providing further structural information on the LcrV-TLR2 complex, we attempted to apply bioinformatical methods to predict the interaction between $Y$. pestis LcrV and $H$. sapiens TLR2. A heterotetrameric model was constructed and evaluated by molecular dynamic simulations in an aquatic system. Based on this structural model, we are able to predict structural contacts between LcrV and TLR2, and identify key regions essential for LcrV function. A model on the mechanism by which $\mathrm{LcrV}$ regulates immune response is raised.

\section{Results}

Modelling and assessment of the LcrV-TLR2 complex structure

Two X-ray diffraction structures (PDB ID: 1R6F and 4JBU) were previously reported for mutants of $Y$. pestis LcrV. The $2.17 \AA$ 1R6F structure mutated $\mathrm{KDK}_{40-42}$ to AAA, deleted $\mathrm{Y}_{90}$, lacked $D_{51}$ to $\mathrm{N}_{51}$ and $\mathrm{N}_{263}$ to $C_{273}$. The $1.65 \AA$ 4JBU structure lacked $\mathrm{N}_{263}$ to $\mathrm{P}_{279}$. These flaws were fixed by performing homologous modelling of the $G_{28}-D_{322}$ fragment of $Y$. pestis LcrV (Uniprot accession P0C7U7, full length $326 \mathrm{AA}$ ) using these two reported structures as templates. A similar approach was done to obtain the modelled structure of TLR2 extracellular domain (Uniprot accession O60603) based on the previously reported H. sapiens-hagfish fusion TLR2 structure (PDB ID 2Z7X) and Mus musculus TLR2 structure (PDB ID 5D3I). The modelled LcrV and TLR2 structures were evaluated to confirm their quality (Additional file 1). The heterotetrameric LcrV-TLR2 complex structure model was subsequently constructed by consecutive modelling the LcrV dimeric structure, LcrVTLR2 heterodimeric structure, and ultimately the LcrVTLR2 heterotetrameric structure.

The stability of the LcrV-TLR2 heterotetrameric structure model was assessed by performing molecular dynamics analysis of the structure in water environments over a time frame of $100 \mathrm{~ns}$ and time interval of $10 \mathrm{ps}$

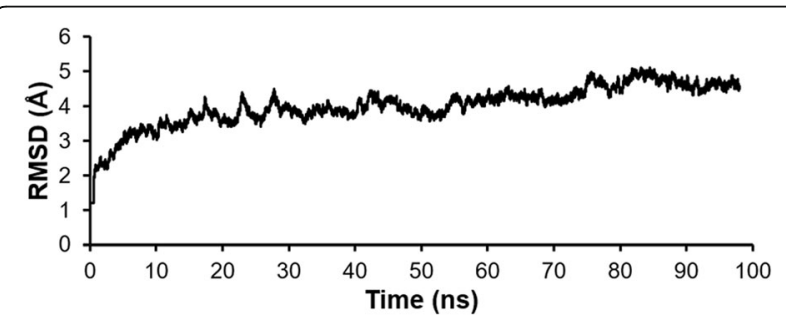

Fig. 1 Stability of modelled LcrV-TLR2 complex structure in water environment 
(Fig. 1). The RMSD of the structure (in comparison with the modelled heterotetrameric structure) stabilized after 20 ns, reaching approximately $5 \AA$ at the end of 100 ns. This assessment suggests that the LcrV-TLR2 heterotetrameric structure model in a water environment is stable, confirming the quality of the structure.

\section{Overall structure of the modelled LcrV-TLR2 complex}

The overall modelled structure of the LcrV-TLR2 complex is a heterotetramer formed by two $Y$. pestis LcrV subunits and two $H$. sapiens TLR2 subunits (Fig. 2a). The modelled LcrV monomer has a unique dumbbellshape structure: two globule modules connected by a long coiled-coil structure formed by two long antiparallel $\alpha$ helices (Fig. 2b), in consistence with previously solved crystal structures of LcrV. The three modules are respectively termed Domain G1 (membrane-adjacent globule), CCL (coiled-coil linker), and G2 (loop-rich globule away from membrane). Domain G1 in modelled LcrV structure is formed by six $\alpha$-helices, of which $\alpha 1$ and $\alpha 2$ are connected by a long loop. Domain G2 is a loop-rich globule module stabilized with two antiparallel $\beta$ strand pairs and four short $\alpha$ helices. A $\beta$-hairpin structure connects $\alpha 7$ and $\alpha 8$, while a long loop connects $\alpha 11$ and $\alpha 12$ in the modelled structure.

In this modelled LcrV-TLR2 heterotetramer, two horseshoelike TLR2 subunits form a V-shaped structure with a dihedral angle of approximately 70 degrees, with their openings facing towards the membrane. The two LcrV structures are sandwiched between the two TLR2 subunits in this model (Fig. 2a). The two TLR2 subunits have very few direct contacts in the model. Instead, they were held together by the two LcrV subunits, forming a LcrV-TLR2 heterotetrameric complex.
Proposed basis for LcrV-TLR2 heterotetramer formation Analysis of the modelled LcrV-TLR2 heterotetramer leads to the proposal that the dimeric LcrV structure is formed via the contacts in primarily Domain G2 and CCL. The extended loop region between $\beta 1$ and $\alpha 2$ in Domain G1 ( $\mathrm{YDP}_{50-52}$ and EVFA $\left.{ }_{57-60}\right)$ could form contacts between the two monomers, potentially by $\pi-\pi$ stacking between $\mathrm{Y}_{50}$. The two $\alpha 7$ in Domain CCL in each monomer form close contacts, and are potentially held together by hydrogen bonds between $R_{150}$ and $S_{151}$ (4.1 $\AA$ ), as well as between $R_{154}$ and $E_{155}(3.0-4.1 \AA)$. The $\alpha 9$ (GYTDEEIFKA $\left.A_{200-209}\right)$ of Domain $G 2$ forms close contacts with $\alpha 12$ (SDITSRKNSAIEA $292-304$ ) of Domain CCL. This contact is formed via a hydrogen bond network: the hydroxyl group of $\mathrm{Y}_{201}$ (donor) forms a hydrogen bond with the side chain carboxamide of $\mathrm{N}_{299}$ (acceptor, 2.6-3.8 $\AA$ ); while the hydroxyl group of $\mathrm{S}_{300}$ forms hydrogen bonds with the peptidyl carbonyl group (acceptor) of $\mathrm{A}_{209}(2.9 \AA), \mathrm{I}_{206}(2.6 \AA)$, and $\mathrm{E}_{205}$ (3.0 ̊) (Fig. 3). Interestingly, the peptidyl carbonyl group (donor) of $S_{300}$ forms hydrogen bonds with the peptidyl amino group (acceptor) of $\mathrm{I}_{302}(3.2 \AA), \mathrm{E}_{303}(3.1 \AA)$, and $A_{304}$ (3.2), suggesting the key role of this residue in the formation of the hydrogen bond network for intact dimeric structure formation.

Further analysis of the heterotetrameric LcrV-TLR2 structure model suggests both LcrV subunits potentially form contacts with each TLR2 subunit. The LcrV subunit on the 'same side' shows extensive contacts with TLR2 in all three domains in the model. A total of 20 hydrogen bonds are formed between Domain G1 and TLR2 (Table 1). These hydrogen bonds form a network that fits Domain G1 in the hollow center of the horseshoe like TLR2 structure. In particular, two regions,
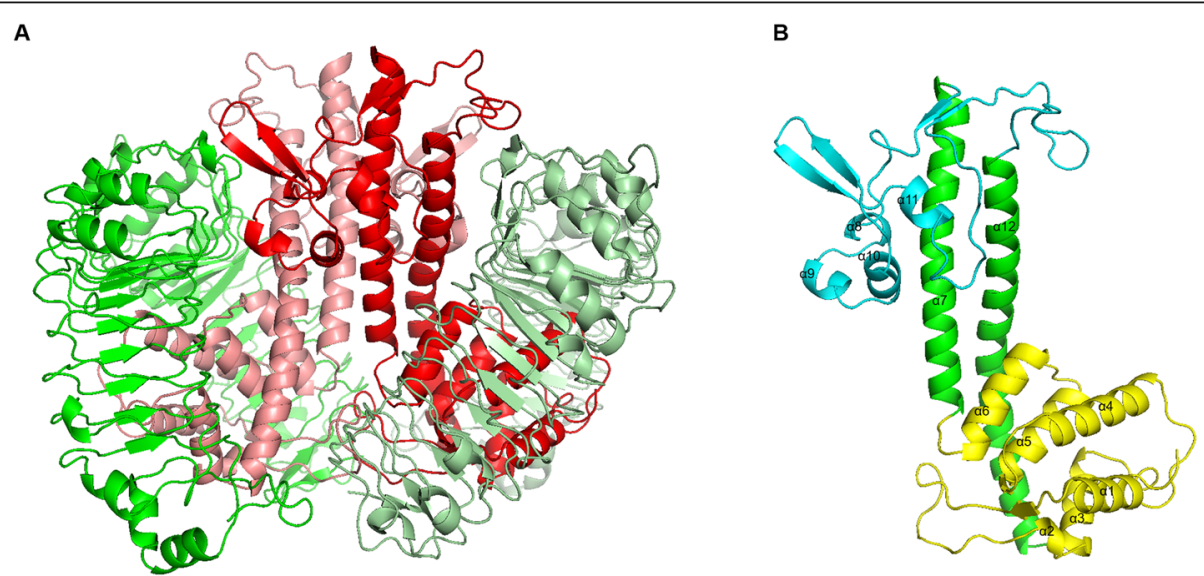

Fig. 2 Modelled structures of $Y$. pestis LCrV and the LCrV-TLR2 heterotetramer. Panel (a), LCrV-TLR2 complex structure model, shown in red colors are LcrV subunits, shown in green colors are TLR2 periplasmic fragments; Panel (b), LcrV monomer, yellow color indicates the N-terminal globule module (Domain G1), blue color indicates the C-terminal globule module (Domain G2), green color indicates the coiled-coil structure connecting the two globule modules (Domain CCL), a helices are indicated 


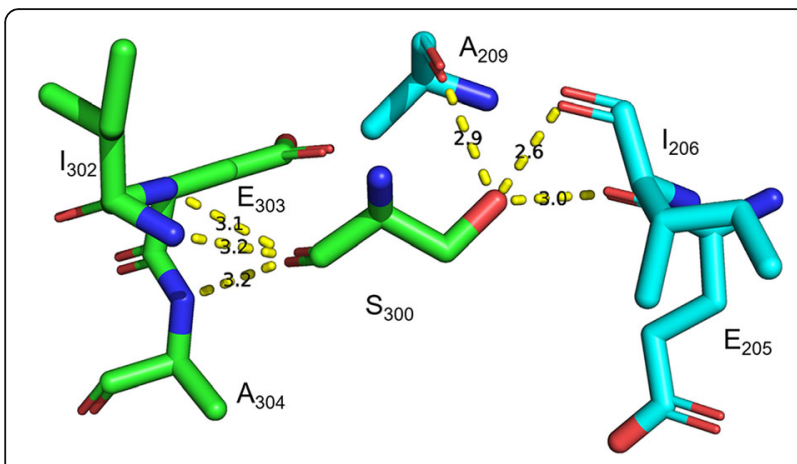

Fig. 3 Hydrogen bonds formed by $S_{300}$ in structure model. Dashed lines indicate potential hydrogen bonds. Numbers indicate bond length (in A). Green and light blue color backbones indicate two different LcrV monomers. Blue color indicates nitrogen atoms. Red color indicates oxygen atoms

namely ADRIDD ${ }_{128-133}$ and $\mathrm{H}_{145} \mathrm{H}_{146}$, are two hubs for hydrogen bond formation and may play key roles in the binding of LcrV and TLR2 (Fig. 4a). Two additional interactions are also involved in the binding of Domain G1 and TLR2: the cation- $\pi$ interaction between LcrV $\mathrm{N}_{92}$ and TLR2 $\mathrm{Y}_{364}$ (Fig. $4 \mathrm{~b}$ ), as well as the $\pi-\pi$ interaction between LcrV $Y_{77}$ and TLR2 $D_{557}$ (Fig. 4c). The

Table 1 Predicted hydrogen bonds between LcrV Domain G1 and TLR2 on the same side

\begin{tabular}{|c|c|c|c|}
\hline LcrV residue & LcrV secondary structure & TLR2 residue & Bond length $(\AA)$ \\
\hline$\overline{N_{43}}$ & Linker between a1 and $\beta 1$ & $E_{103}$ & 3.0 \\
\hline $\mathrm{Q}_{93}$ & $a 4$ & $Y_{364}$ & 4.0 \\
\hline $\mathrm{N}_{96}$ & $a 4$ & $\mathrm{R}_{340}$ & 3.0 \\
\hline$K_{99}$ & $a 4$ & $K_{253}$ & 3.5 \\
\hline$R_{100}$ & $a 4$ & $R_{315}$ & 3.6 \\
\hline$E_{106}$ & $a 4$ & $E_{178}$ & 3.0 \\
\hline$Q_{112}$ & Linker between $\beta 2$ and a5 & $K_{37}$ & 2.6 \\
\hline$A_{128}$ & Loop between a5 and a6 & $\mathrm{R}_{395}$ & 2.8 \\
\hline$A_{128}$ & Loop between a5 and a6 & $\mathrm{Q}_{396}$ & 2.8 \\
\hline$A_{128}$ & Loop between a5 and a6 & $K_{422}$ & 2.8 \\
\hline$R_{130}$ & Loop between a5 and a6 & $\mathrm{R}_{315}$ & 3.0 \\
\hline$R_{130}$ & Loop between a5 and a6 & $E_{344}$ & 3.5 \\
\hline$D_{132}$ & Loop between a5 and a6 & $R_{316}$ & 3.9 \\
\hline$D_{133}$ & a6 & $\mathrm{R}_{257}$ & 3.3 \\
\hline$K_{137}$ & $a 6$ & $Y_{109}$ & 4.2 \\
\hline $\mathrm{H}_{145}$ & a6 & $\mathrm{S}_{39}$ & 3.8 \\
\hline $\mathrm{H}_{145}$ & $a 6$ & $\mathrm{D}_{58}$ & 2.7 \\
\hline $\mathrm{H}_{145}$ & a6 & $S_{60}$ & 3.3 \\
\hline $\mathrm{H}_{146}$ & Linker between a6 and a7 & $N_{61}$ & 3.2 \\
\hline $\mathrm{H}_{146}$ & Linker between a6 and a7 & $\mathrm{S}_{40}$ & 3.0 \\
\hline
\end{tabular}

LcrV Domain G2 loop region between $\beta 6$ and $\alpha 12$, namely $\mathrm{ELS}_{265-267}$ and a histidine derivative at position 268 , is another key location for the binding to TLR2 due to the hydrogen bond network between these residues and TLR2 (Fig. 5). In LcrV Domain CCL, $\alpha 12$ forms an extensive hydrogen bond network with TLR2, with 19 predicted hydrogen bonds formed (Table 2).

The LcrV subunit on the 'opposite side' also forms close contacts with TLR2 subunit in the model, reinforcing the LcrV-TLR2 heterotetramer formation. Three regions are involved in the interaction between the 'opposite side' LcrV and TLR2: the loop region between $\beta 1$ and $\alpha 2$ in Domain G1, the $\alpha 8-\alpha 9$ linker and the beginning of $\alpha 9$ in Domain G2, as well as a $\mathrm{Q}_{317}$ residue in $\alpha 12$ of Domain CCL (Table 3). Interestingly, TLR2

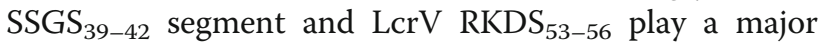
role in forming this hydrogen bond network, and are involved in 8/13 hydrogen bonds formed (Fig. 6).

\section{A structure-based mechanistic model for LcrV-TLR2 complex formation and the role of LcrV in immune response}

From the modelled heterotetrameric structure of LcrVTLR2 complex, a model for the role of each LcrV domain could be proposed (Fig. 7a). In the LcrV-TLR2 structure model, the formation of LcrV dimer is primarily due to the extensive interactions between CCL domains and the $\alpha 8$-containing loop region of Domain G2 $\left(\mathrm{Y}_{201}-\mathrm{A}_{209}\right)$. The two TLR2 subunits are not directly associated in the structure model. Instead, they are held together via extensive interactions with both LcrV subunits. Several regions were found essential for the formation of the heterotetrameric complex in the structure model: the $\beta$ strand containing loop (43-63) in Domain G1, $\alpha 4(92-107)$ in Domain G1, $\alpha 6$ and its linker to $\alpha 5$ (127-145) in Domain G1, the whole CCL domain, the loop region on the N-terminus of $\alpha 12$, and $\alpha 8$ containing loop region of Domain G2 (196-208).

One prominent phenomenon we observed in the LcrV-TLR2 complex structure model is that the LcrV subunits separate the two TLR2 subunits in the complex. In this configuration, the TIR-connecting $\mathrm{C}$-terminus of TLR2 extracellular section were separated by two LcrV subunits, making it impossible for the formation of TIR dimers (Fig. 7b). Therefore, we propose that LcrV functions in inhibiting the immune response of white blood cells by inhibiting TIR dimer formation, the signal transduction via TLR2, and subsequent induction of inflammation factors such as TNF- $\alpha$ [25]. The formation of LcrV-TLR2 complex also competitively inhibits the binding of other toll-like receptors (such as TLR1 and TLR6) with TLR2 for immune response. A model of LcrV in immune response can be summarized in Fig. 7c. 
A

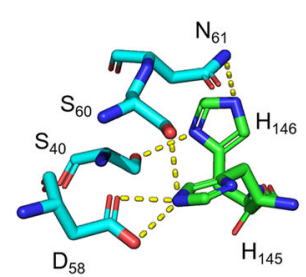

B

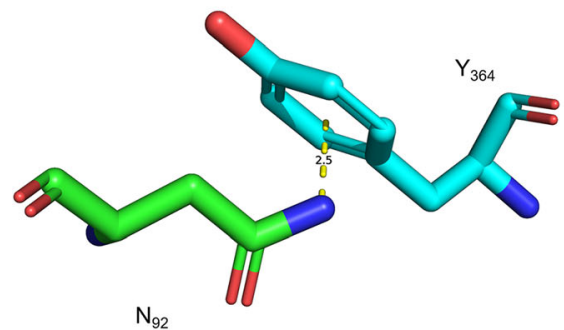

C
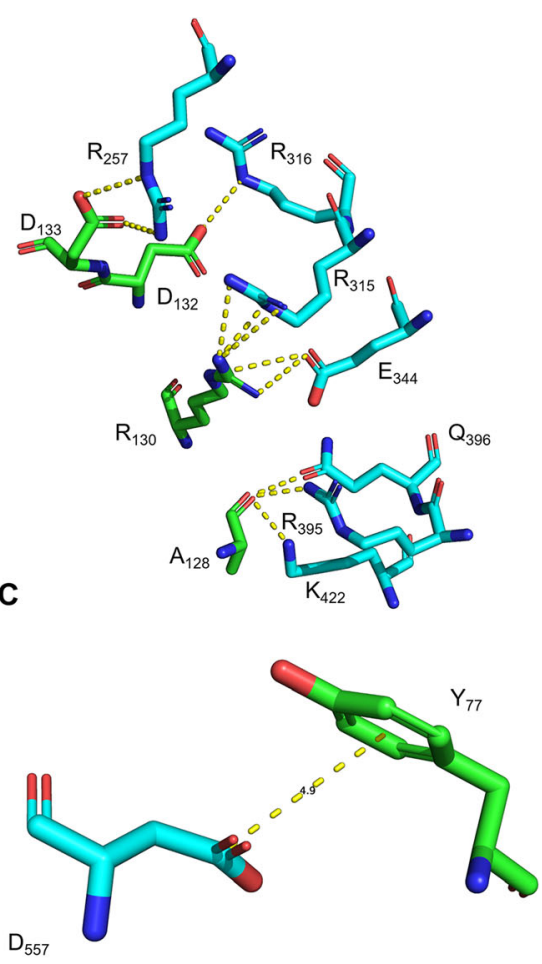

Fig. 4 Proposed interactions between TLR2 and LcrV Domains G1/CCL on the same side. Panel (a): hydrogen bond network, dashed lines indicate potential hydrogen bonds; Panel (b), cation- $\pi$ interaction between LCrV $N_{92}$ and TLR2 $Y_{364}$; Panel (c), $\pi-\pi$ interaction between LcrV $Y_{77}$ and TLR2 $D_{557}$. Green and light blue color backbones respectively indicate TLR2 and LcrV. Blue color indicates nitrogen atoms. Red color indicates oxygen atoms. Numbers indicate bond length (in $\AA$ )

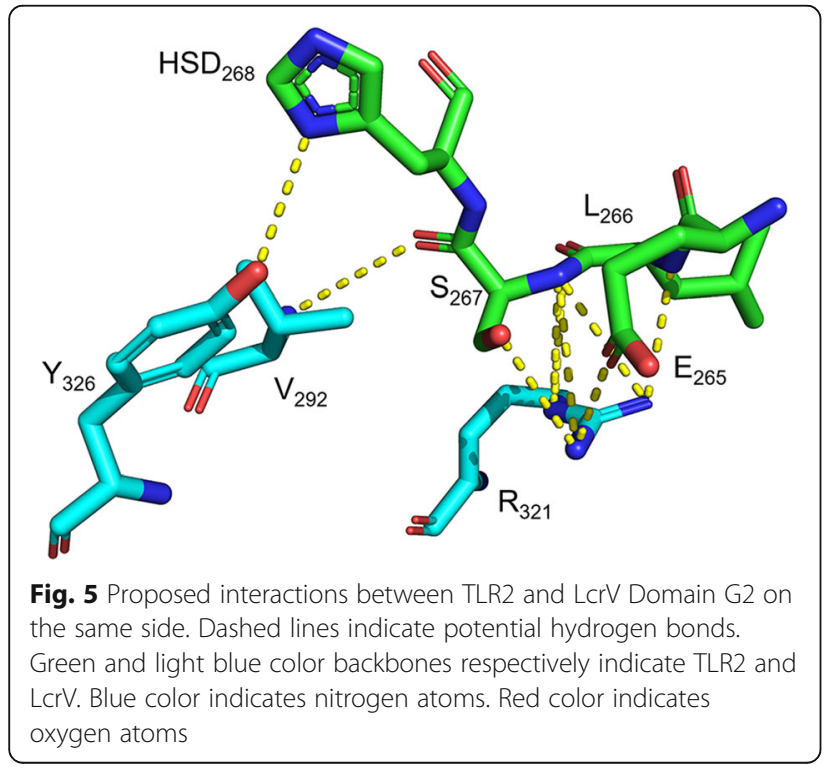

\section{Discussion}

A large body of literature discussed $Y$. pestis LcrV and its immunological repression function involving H. sapiens TLR2 and other proteins [1, 17-22, 24], yet the mechanistic insights on how LcrV binds to TLR2 for its function have been under controversy due to the lack of a LcrV-TLR2 complex structure. In this work, with a modelling-based approach, we successfully obtained a LcrV-TLR2 heterotetrameric complex structure model, from which a mechanistic model for the function of LcrV was proposed.

In this model, LcrV functions in spatially separating the two TLR2 subunits to prevent the formation of functional TIR dimers. LcrV may also recruit TLR2 and competitively prevent the formation of functional complexes of TLR2 and other TLR subunits. This model explains why $\mathrm{D}_{203}-\mathrm{I}_{206}$ and $\mathrm{T}_{271}-\mathrm{S}_{300}$ are so important in the function of $\operatorname{LcrV}[19,20]$ : the former segment is the key to the binding of LcrV to LcrV, while the later segment is essential for the binding of LcrV and TLR2 [19]. The deletion of $\mathrm{D}_{203}-\mathrm{I}_{206}$ reduces the function of LcrV but cannot totally abolish it, as Domain G2 also helps the formation of LcrV dimer (Fig. 7a). However, the removal of $\mathrm{T}_{271}-\mathrm{S}_{300}$ not only removed the largest surface for LcrV-TLR2 interaction, but may also lead to 
Table 2 Predicted hydrogen bonds between LcrV Domain CCL and TLR2 on the same side

\begin{tabular}{|c|c|c|c|}
\hline LcrV residue & LcrV secondary structure & TLR2 residue & Bond length $(\AA)$ \\
\hline \multirow[t]{2}{*}{$\mathrm{D}_{294}$} & a12 & $\mathrm{H}_{318}$ (two nitrogen atoms on side chain imidazole group) & 3.0 \\
\hline & & & 3.1 \\
\hline \multirow[t]{3}{*}{$\mathrm{D}_{294}$} & a12 & $\mathrm{R}_{316}$ (three side chain amino groups) & 2.6 \\
\hline & & & 2.8 \\
\hline & & & 3.1 \\
\hline \multirow[t]{2}{*}{$R_{297}$} & $a 12$ & $\mathrm{R}_{316}$ (two side chain amino groups) & 2.6 \\
\hline & & & 3.0 \\
\hline$R_{297}$ & a12 & $D_{286}$ & 4.0 \\
\hline$K_{311}$ & $a 12$ & $\mathrm{R}_{486}$ & 3.7 \\
\hline$R_{318}$ & a12 & $G_{532}$ (peptidyl carbonyl group) & 3.6 \\
\hline$R_{318}$ & a12 & $\mathrm{G}_{532}$ (peptidyl amino group) & 3.2 \\
\hline$L_{320}$ & $a 12$ & $Q_{574}$ & 3.4 \\
\hline$D_{321}$ & a12 & $\mathrm{Q}_{574}$ & 3.4 \\
\hline$D_{321}$ & a12 & $N_{561}$ & 3.5 \\
\hline$D_{321}$ & a12 & $Y_{562}$ & 3.9 \\
\hline$D_{321}$ & $a 12$ & $\mathrm{~L}_{563}$ & 3.5 \\
\hline \multirow[t]{2}{*}{$\mathrm{D}_{322}$ (two side chain carbonyl groups) } & $a 12$ & $N_{561}$ & 3.1 \\
\hline & & & 3.3 \\
\hline$D_{322}$ & $a 12$ & $W_{558}$ & 3.8 \\
\hline
\end{tabular}

significant change of Domain G1 structure, leading to the inability of LcrV to bind to TLR2, agreeing to previous findings [20].

The most striking feature of the structure model of LcrV-TLR2 is the extent of interactions involved in the maintenance of the structure. In addition to previously found key regions for function, as shown in Fig. 7a, all three domains of LcrV are involved in the binding between LcrV to LcrV, and LcrV to TLR2. These extensive interactions make the binding of LcrV to TLR2 resistance to mutation: minor mutations, even in critical binding regions, do not change the overall binding of LcrV and TLR2, and subsequently the effectiveness of LcrV. This feature makes it particularly difficult for host cells to resist LcrV, and $Y$. pestis invasion. Recent investigations showed

Table 3 Predicted hydrogen bonds between LcrV and TLR2 on the opposite side

\begin{tabular}{llll}
\hline LcrV residue & LcrV secondary structure & TLR2 residue & Bond length $(\AA)$ \\
\hline$R_{53}$ & Loop between $\beta 1$ and a2 & 2.6 \\
$K_{54}$ (peptidyl carbonyl group) & Loop between $\beta 1$ and a2 & $S_{40}$ & 2.6 \\
$K_{54}$ (peptidyl amino group) & Loop between $\beta 1$ and a2 & $S_{40}$ & 4.0 \\
$K_{54}$ & Loop between $\beta 1$ and a2 & $S_{40}$ & 3.1 \\
$D_{55}$ & Loop between $\beta 1$ and a2 & $G_{41}$ & 4.1 \\
$S_{56}$ & Loop between $\beta 1$ and a2 & $S_{40}$ & 3.4 \\
$S_{56}$ & Loop between $\beta 1$ and a2 & $S_{27}$ & 3.9 \\
$S_{56}$ (side chain hydroxyl group) & Loop between $\beta 1$ and a2 & $S_{39}$ & 2.6 \\
$S_{56}$ (peptidyl amino group) & Loop between $\beta 1$ and a2 & $S_{40}$ & 3.2 \\
$E_{57}$ & Loop between $\beta 1$ and a2 & $S_{40}$ & 3.1 \\
$T_{202}$ & Linker between a8 and a9 & $S_{29}$ & 3.2 \\
$E_{205}$ & a9 & $H_{318}$ & 3.8 \\
$Q_{307}$ & a12 & $\mathrm{Q}_{345}$ & 3.9 \\
\hline
\end{tabular}




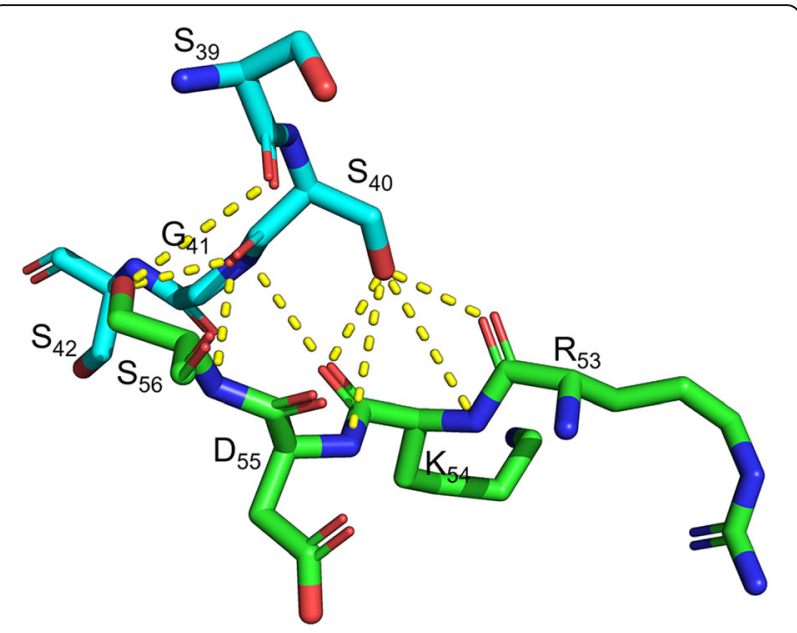

Fig. 6 Proposed hydrogen bond network between TLR2 SSGS $39-42$ and LCrV RKDS ${ }_{53-56}$ on the opposite side. Dashed lines indicate potential hydrogen bonds. Green and light blue color backbones respectively indicate TLR2 and LcrV. Blue color indicates nitrogen atoms. Red color indicates oxygen atoms

the amino acid polymorphism in Yersinia LcrV proteins that enables immune escape [26, 27]. Interestingly, only one of the variable sites $\left(\mathrm{E}_{205}\right)$ is involved in hydrogen bond formation, implicating the importance of this hydrogen bond network between LcrV monomers and between LcrV/TLR2 for its function.

In previous biochemical and immunological work, controversies stood on the mechanism of LcrV function: although research generally agreed that LcrV represses immunological factors such as TNF- $\alpha$, whether this repression is mediated by stimulating IL10 has been controversial $[21,22]$. The mechanistic model established in this work supports the repression of TNF- $\alpha$ by LcrV as binding of LcrV with TLR2 prevents TIR dimers formation, therefore blocking TNF- $\alpha$ stimulation (Fig. 7c). The stimulation of IL-10, on the other hand, was not shown in this proposed model. Therefore, whether IL-10 stimulation is involved in the function of LcrV remains unknown, and further investigation is required to determine the role of IL-10.

Previous research showed large multimers of LcrV (> $200 \mathrm{kD}$ ) can stimulate TLR2 leading to IL-8 formation [21]. We suspect this stimulation is due to the formation of large $\operatorname{LcrV}_{2 \mathrm{n}}-\mathrm{TLR}_{2 \mathrm{n}}$ aggregates which brings TLR2 moieties from different LcrV-TLR2 heterotetramers in close proximity, leading to immune response.

In addition to the regions proposed to be involved in LcrV-TLR2 complex formation, the role of the potentially active hairpin $\left(\mathrm{P}_{220}-\mathrm{I}_{232}\right)$ structure in Domain G2 remains to be elucidated. Previous report showed that CD14 is involved in the interaction between LcrV and
TLR2 [17]. We suspect that this region functions in binding to CD14 or other functional molecules for complete activity of LcrV-TLR2 complex.

\section{Conclusions}

In conclusion, a structural model of the $Y$. pestis LcrV-H. sapiens TLR2 complex was constructed. The modelled structure is a $\mathrm{LcrV}_{2}-\mathrm{TLR} 2_{2}$ heterotetramer. Analysis of the structure model revealed that the TLR2 subunits are held together by interactions between the two LcrV monomers and LcrV-TLR2 interactions. A mechanistic model was constructed from the modelled structure: The LcrV dimer separates the TLR2 subunits upon binding, leading to separation of the TIR domains linked at the C-terminus of TLR2 extracellular domain, thereby abolishing immune response; LcrV also binds to TLR2 and competitively prevents the formation of functional heterodimers of TLR2 and other TLRs. This model explains previous experimental phenomenon, and reveals more sites essential for the function of LcrV.

\section{Methods}

Modelling of protein structures and structure evaluation

The modelling of $Y$. pestis LcrV and $H$. sapiens TLR2 structures was performed using previously reported LcrV mutant structures (PDB ID: 4JBU, 1R6F) and $H$. sapiens-hagfish TLR2 fusion/M. musculus TLR2 protein structures (PDB ID: 2Z7X, 5D3I) as templates $[1,24,25,28,29]$, and native $Y$. pestis $\mathrm{LcrV} / H$. sapiens TLR2 sequences (Uniprot accession P0C7U7 and O60603). Modelling was performed using I-TASSER, SWISS-MODEL or Modeller [30-32]. Modelled structures were evaluated using ProQ, Verify3D, Procheck, Modfold, and QMEAN [33-37]. The best model was chosen for further optimization of the loop region using Modloop [38]. The final modelled structure is shown in Additional file 2.

\section{Modelling of LcrV-TLR2 complex structure}

The structures of LcrV homodimer and LcrV-TLR2 heterodimers were modelled using GrammX [39]. The LcrV-TLR2 heterotetramer structure was constructed by manually matching LcrV in LcrV homodimers to LcrVTLR2 heterodimers.

\section{Molecular dynamics simulation}

Molecular dynamics simulation of the modelled LcrVTLR2 structure in water environment was performed using the Nanoscale Molecular Dynamics program (NAMD) that was developed by the Theoretical and Computational Biophysics Group in the Beckman Institute for Advanced Science and Technology at the 


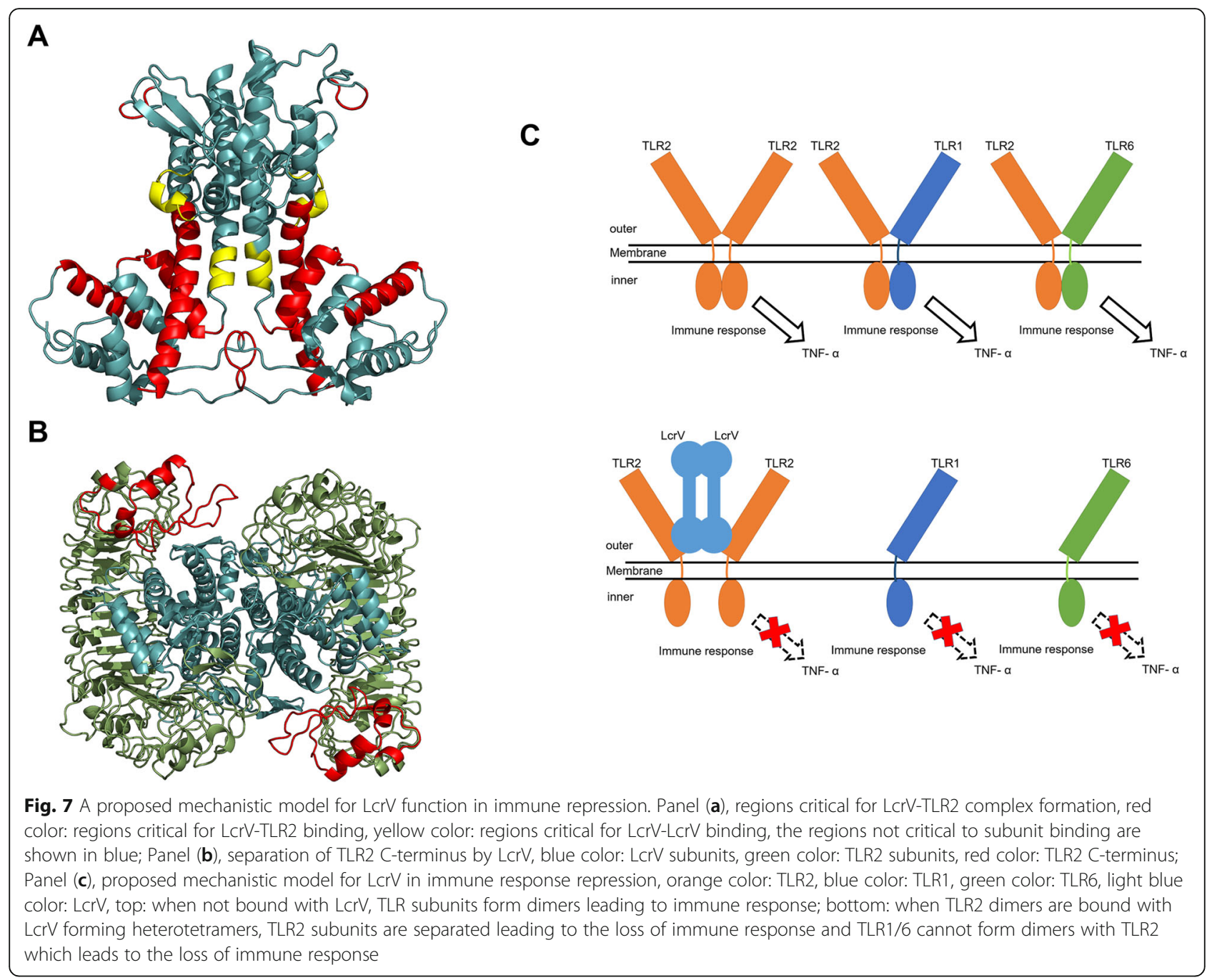

University of Illinois at Urbana-Champaign (http:// www.ks.uiuc.edu/Research/namd/) [40].

\section{Protein structure visualization and measurement}

Protein structure visualization and measurement of distances/dihedral angle was performed using the PyMOL Molecular Graphics System version 2.2.3.

\section{Supplementary information}

Supplementary information accompanies this paper at https://doi.org/10. 1186/s12865-019-0329-5.

Additional file 1. Quality evaluation of modelled structures.

Additional file 2. Modelled structure of LcrV-TLR2 heterotetrameric complex.

\section{Abbreviations}

CCL: Coiled-coil linker; LcrV: Low Calcium Response V; NAMD: Nanoscale Molecular Dynamics program; T3SS: Type III Secretion System; TLR2: Toll-like Receptor 2; Yop: Yersinia outer membrane protein

\section{Acknowledgements}

We would like to thank the core facilities for life and environmental sciences in Shandong University for technical assistance.

\section{Authors' contributions}

TD, JG and GQ performed bioinformatical analysis; TD, JG, MW, and HX interpreted the data; TD, JG and MW wrote the manuscript; All authors critically revised the manuscript, read and approved the final manuscript.

\section{Funding}

This work was supported by the National Natural Science Foundation of China (31501064, 31770042, 31770043), the National Key Research and Development Program of China (2017YFD0400301), Shandong Province Key Research and Development Program (2016GSF121040, 2018GSF118008), the Fundamental Research Funds of Shandong University (2017JC028, 2018JC013, 2018JC027), the State Key Laboratory of Microbial Technology Open Project Funds, Shandong University (M2018-07), and Jinan Cultural Industry Development Fund.

The funding bodies have no roles in the design of the study; collection, analysis, and interpretation of data; and in writing the manuscript.

Availability of data and materials

The datasets used and/or analyzed during the current study are available from the corresponding author on reasonable request. 
Ethics approval and consent to participate

Not applicable.

\section{Consent for publication}

Not applicable.

\section{Competing interests}

The authors declare that they have no competing interests.

\section{Author details}

'State Key Laboratory of Microbial Technology, Microbial Technology Institute, Shandong University, Qingdao, China. ${ }^{2}$ Taishan College, Shandong University, Qingdao, China. ${ }^{3}$ School of Life Sciences, Shandong University, Qingdao, China.

Received: 1 April 2019 Accepted: 26 November 2019 Published online: 16 December 2019

\section{References}

1. Derewenda U, Mateja A, Devedjiev Y, Routzahn KM, Evdokimov AG, Derewenda $\mathrm{ZS}$, et al. The structure of Yersinia pestis $\mathrm{V}$-antigen, an essential virulence factor and mediator of immunity against plague. Structure. 2004;12:301-6.

2. Perry RD, Fetherston JD. Yersinia pestis - etiologic agent of plague. Clin Microbiol Rev. 1997;10:35-66.

3. Stenseth NC, Atshabar BB, Begon M, Belmain SR, Bertherat E, Carniel E, et al. Plague: past, present, and future. PLoS Med. 2008;5:e3.

4. Gur D, Glinert I, Aftalion M, Vagima Y, Levy Y, Rotem S, et al. Inhalational gentamicin treatment is effective against pneumonic plague in a mouse model. Front Microbiol. 2018;9:741.

5. Zhou D, Han Y, Yang R. Molecular and physiological insights into plague transmission, virulence and etiology. Microbes Infect. 2006;8:273-84.

6. Heesemann J, Sing A, Trülzsch K. Yersinia's stratagem: targeting innate and adaptive immune defense. Curr Opin Microbiol. 2006;9:55-61.

7. Hentschke M, Trülzsch K, Heesemann J, Aepfelbacher M, Ruckdeschel K. Serogroup-related escape of Yersinia enterocolitica YopE from degradation by the ubiquitin-proteasome pathway. Infect Immun. 2007:75:4423-31.

8. Cornelis GR. The Yersinia Ysc-Yop 'Type III' weaponry. Nat Rev Mol Cell Biol. 2002;3:742-53.

9. Viboud Gl, Bliska JB. Yersinia outer proteins: role in modulation of host cell signaling responses and pathogenesis. Annu Rev Microbiol. 2005;59:69-89.

10. Brubaker RR. Interleukin-10 and inhibition of innate immunity to Yersiniae: roles of Yops and LcrV (V antigen). Infect Immun. 2003;71:3673-81.

11. Schubert S, Rakin A, Heesemann J. The Yersinia high-pathogenicity island (HPI): evolutionary and functional aspects. Int J Med Microbiol. 2004;294:83-94.

12. Revell PA, Miller VL. Yersinia virulence: more than a plasmid. FEMS Microbiol Lett. 2006;205:159-64.

13. Matson JS, Nilles ML. LcrG-LcrV interaction is required for control of Yops secretion in Yersinia pestis. J Bacteriol. 2001;183:5082-91.

14. Holmström A, Olsson J, Cherepanov P, Maier E, Nordfelth R, Pettersson J, et al. LcrV is a channel size-determining component of the Yop effector translocon of Yersinia. Mol Microbiol. 2001;39:620-32.

15. Motin VL, Nakajima R, Smirnov GB, Brubaker RR. Passive immunity to yersiniae mediated by anti-recombinant $V$ antigen and protein $A-V$ antigen fusion peptide. Infect Immun. 1994;62:4192-210.

16. Nedialkov YA, Motin VL, Brubaker RR. Resistance to lipopolysaccharide mediated by the Yersinia pestis $V$ antigen-polyhistidine fusion peptide: amplification of interleukin-10. Infect Immun. 1997;65:1196-203.

17. Sing A, Rost D, Tvardovskaia N, Roggenkamp A, Wiedemann A, Kirschning $C$, et al. Yersinia $\mathrm{V}$-antigen exploits toll-like receptor 2 and CD14 for interleukin 10-mediated immunosuppression. J Exp Med. 2002;196:1017-24.

18. Sing A, Reithmeier-Rost D, Granfors K, Hill J, Roggenkamp A, Heesemann J. A hypervariable $\mathrm{N}$-terminal region of Yersinia LcrV determines toll-like receptor 2-mediated IL-10 induction and mouse virulence. Proc Natl Acad Sci U S A. 2005;102:16049-54.

19. Abramov VM, Khlebnikov VS, Vasiliev AM, Kosarev IV, Vasilenko RN, Kulikova $\mathrm{NL}$, et al. Attachment of LcrV from Yersinia pestis at dual binding sites to human TLR-2 and human IFN- $\gamma$ receptor. J Proteome Res. 2007;6:2222-31.

20. Overheim KA, DePaolo RW, Debord KL, Morrin EM, Anderson DM, Green $\mathrm{NM}$, et al. LcrV plague vaccine with altered immunomodulatory properties. Infect Immun. 2005;73:5152-9.
21. Pouliot K, Pan N, Wang S, Lu S, Lien E, Goguen JD. Evaluation of the role of LcrV-toll-like receptor 2-mediated immunomodulation in the virulence of Yersinia pestis. Infect Immun. 2007;75:3571-80.

22. Reithmeier-Rost D, Hill J, Elvin SJ, Williamson D, Dittmann S, Schmid A, et al. The weak interaction of LCrV and TLR2 does not contribute to the virulence of Yersinia pestis. Microbes Infect. 2007;9:997-1002.

23. Tito MA, Miller J, Walker N, Griffin KF, Diane Williamson E, Despeyroux-Hill D, et al. Probing molecular interactions in intact antibody: antigen complexes, an electrospray time-of-flight mass spectrometry approach. Biophys J. 2001;81:3503-9.

24. Chaudhury S, Battaile KP, Lovell S, Plano GV, De Guzman RN. Structure of the Yersinia pestis tip protein LcrV refined to $1.65 \AA$ resolution. Acta Crystallogr Sect F Struct Biol Cryst Commun. 2013;F69:477-81.

25. Tao X, Xu Y, Zheng Y, Beg AA, Tong L. An extensively associated dimer in the structure of the C713S mutant of the TIR domain of human TLR2. Biochem Biophys Res Commun. 2002;299:216-21.

26. Daniel C, Dewitte A, Poiret S, Marceau M, Simonet M, Marceau L, et al. Polymorphism in the Yersinia LcrV antigen enables immune escape from the protection conferred by an LcrV-secreting Lactococcus lactis in a pseudotuberculosis mouse model. Front Immunol. 2019;10:1830.

27. Anisimov AP, Dentovskaya SV, Panfertsev EA, Svetoch TE, Kopylov PK, Segelke BW, et al. Amino acid and structural variability of Yersinia pestis LcrV protein. Infect Genet Evo. 2010;10:137-45.

28. Jin MS, Kim SE, Heo JY, Lee ME, Kim HM, Paik SG, et al. Crystal structure of the TLR1-TLR2 heterodimer induced by binding of a tri-acylated lipopeptide. Cell. 2007;130:1071-82.

29. Koymans KJ, Feitsma LJ, Brondijk THC, Aerts PC, Lukkien E, Lössl P, et al. Structural basis for inhibition of TLR2 by staphylococcal superantigen-like protein 3 (SSL3). Proc Natl Acad Sci. 2015;112:11018-23.

30. Webb B, Sali A. Comparative protein structure modeling using MODELLER. Curr Protoc Bioinforma. 2016;54:5.6.1-5.6.37.

31. Waterhouse A, Bertoni M, Bienert S, Studer G, Tauriello G, Gumienny R, et al. SWISS-MODEL: homology modelling of protein structures and complexes. Nucleic Acids Res. 2018;46:W296-303.

32. Yang J, Yan R, Roy A, Xu D, Poisson J, Yang Y. The I-TASSER suite: protein structure and function prediction. Nat Methods. 2015;12:7-8.

33. Wallner B, Elofsson A. Can correct protein models be identified? Protein Sci. 2003:12:1073-86.

34. McGuffin LJ, Buenavista MT, Roche DB. The ModFOLD4 server for the quality assessment of 3D protein models. Nucleic Acids Res. 2013;41:W368-72.

35. Laskowski RA, MacArthur MW, Moss DS, Thornton JM. PROCHECK: a program to check the stereochemical quality of protein structures. J Appl Crystallogr. 1993;26:283-91.

36. Lüthy $R$, Bowie JU, Eisenberg D. Assessment of protein models with threedimensional profiles. Nature. 1992;356:83-5.

37. Benkert $P$, Tosatto SCE, Schomburg D. QMEAN: a comprehensive scoring function for model quality assessment. Proteins Struct Funct Genet. 2008;71:261-77.

38. Fiser A, Do RKG, Šali A. Modeling of loops in protein structures. Protein Sci. 2000:9:1753-73.

39. Tovchigrechko A, Vakser IA. GRAMM-X public web server for protein-protein docking. Nucleic Acids Res. 2006:34:W310-4.

40. Phillips JC, Braun R, Wang W, Gumbart J, Tajkhorshid E, Villa E, et al. Scalable molecular dynamics with NAMD. J Comput Chem. 2005;26:1781-802.

\section{Publisher's Note}

Springer Nature remains neutral with regard to jurisdictional claims in published maps and institutional affiliations.

Ready to submit your research? Choose BMC and benefit from:

- fast, convenient online submission

- thorough peer review by experienced researchers in your field

- rapid publication on acceptance

- support for research data, including large and complex data types

- gold Open Access which fosters wider collaboration and increased citations

- maximum visibility for your research: over $100 \mathrm{M}$ website views per year

At BMC, research is always in progress.

Learn more biomedcentral.com/submissions 